CrossMark < click for updates

Cite this: Phys. Chem. Chem. Phys., 2016, 18, 7535

DOI: $10.1039 / c 6 c p 90050 j$

www.rsc.org/pccp

\section{Correction: Strain effects on oxygen migration in perovskites}

\author{
Tam Mayeshiba $^{a}$ and Dane Morgan ${ }^{\mathrm{b}}$ \\ Correction for 'Strain effects on oxygen migration in perovskites' by Tam Mayeshiba et al., Phys. Chem. \\ Chem. Phys., 2015, 17, 2715-2721.
}

An error in one of our equation of state analysis codes mistaking Python 2.7 integer division for float division produced delta in migration energy per percent strain (DMEPS) values for the elastic strain model that were erroneously small in magnitude. Correcting this error affects all of the discussion and data associated with the elastic strain model, and improves the comparison of the elastic strain model's DMEPS values with the DMEPS values fit to ab initio migration barriers.

The essential plot is corrected below as Fig. 5R, which shows that the DMEPS predicted by the elastic strain model now follow the same trend as our ab initio DMEPS. In contrast to the claim made in the original paper, the strain model DMEPS no longer underrepresent the ab initio DMEPS.

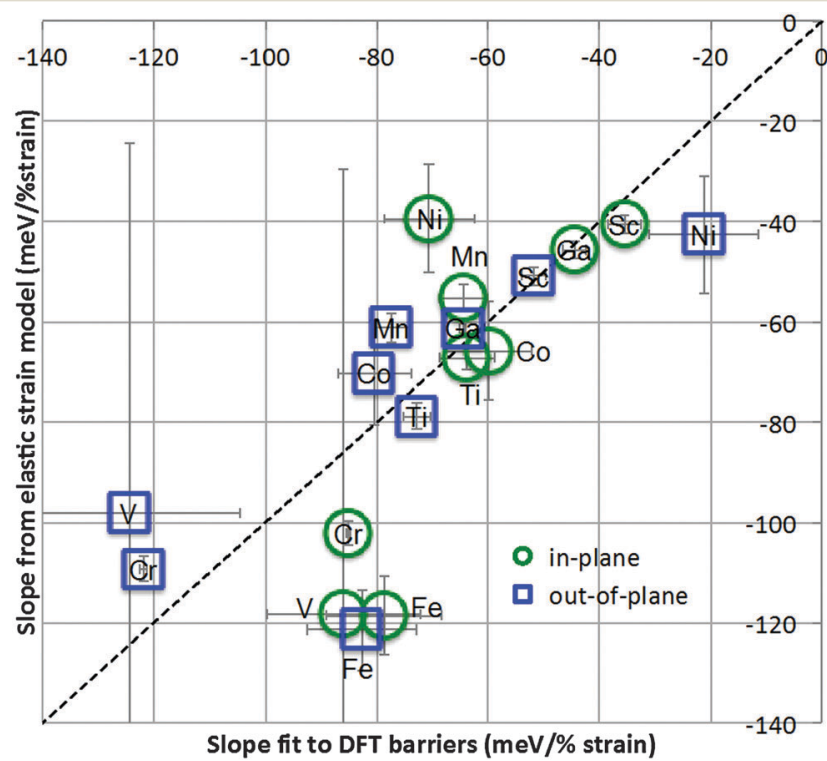

Fig. 5 (R) Elastic formula DMEPS versus fitted DMEPS for migration barriers as a function of strain (dashed line indicates perfect match). Data point is the center of each symbol. Error bars are based on uncertainties in fitting elastic constants and are discussed in the electronic supplementary information (ESI) of the original paper, Section S12. All error bars are symmetric.

The corrected strain model DMEPS differ from the ab initio DMEPS by an average of $16 \pm 13 \mathrm{meV} / \%$ strain (where the uncertainty represents one standard deviation of the error from the mean) with a maximum error of $40 \mathrm{meV} / \%$ strain and

\footnotetext{
${ }^{a}$ Materials Science Program, University of Wisconsin-Madison, Madison, WI, 53706, USA

${ }^{b}$ Department of Materials Science and Engineering, University of Wisconsin-Madison, Madison, WI, 53706, USA. E-mail: ddmorgan@wisc.edu
} 
a root-mean-squared error of $20 \mathrm{meV} / \%$ strain. These values are improved from the average of $22 \pm 16 \mathrm{meV} / \% \mathrm{strain}, \mathrm{maximum}$ error of $56 \mathrm{meV} / \%$ strain, and root-mean-squared error of $27 \mathrm{meV} / \%$ strain in the original paper.

The elastic strain model therefore qualitatively describes the response of oxygen migration barriers in perovskites with respect to biaxial strain, including the correct overall trend. However, the model has significant errors for a number of specific systems, and the discussion of those systems from the original paper remains valid.

The Royal Society of Chemistry apologises for these errors and any consequent inconvenience to authors and readers. 